\title{
Primeros hallazgos de Aedes (Stegomyia) albopictus (Skuse, 1894) en la provincia de Valencia, España
}

Pedro $\mathrm{M}^{\mathrm{a}}$ Alarcón-Elbal ${ }^{1}$, Sarah Delacour-Estrella ${ }^{1,2}$, Francisco Collantes ${ }^{3}$, Juan Antonio Delgado ${ }^{3}$, Ignacio RuizArrondo $^{1,2}$, Rocío Pinal-Prieto ${ }^{1}$, Rosario Melero-Alcíbar ${ }^{4}$, Ricardo Molina ${ }^{4}$, María J. Sierra ${ }^{5}$, Carmen Amela ${ }^{5}$ \& Javier Lucientes ${ }^{1}$

1 Departamento de Patología Animal, Unidad de Parasitología y Enfermedades Parasitarias. Facultad de Veterinaria, Universidad de Zaragoza, España. C/ Miguel Servet 177. 50013 Zaragoza, España.

2 Quimera Biological Systems. Pol. Ind. Malpica - Alfindén, C/ Olivo 14, nave 6. 50171 La Puebla de Alfindén, Zaragoza, España.

3 Departamento de Zoología y Antropología Física, Facultad de Biología, Campus de Espinardo, Universidad de Murcia. 30100 Murcia, España.

4 Servicio de Parasitología, Centro Nacional de Microbiología, Instituto de Salud Carlos III, Ctra. Pozuelo-Majadahonda, km 2. 28220 Majadahonda, Madrid, España.

5 Centro de Coordinación de Alertas y Emergencias Sanitarias. Dirección General de Salud Pública, Calidad e Innovación. Ministerio de Sanidad, Servicios Sociales e Igualdad.

\section{Resumen}

\section{Correspondencia}

P. M. Alarcón-Elbal

E-mail: pedro.alarcon@uv.es

Recibido: 15 octubre 2013

Aceptado: 14 noviembre 2013

Publicado on-line: 15 noviembre 2013
Enmarcado dentro del proyecto "Vigilancia entomológica en aeropuertos y puertos frente a vectores importados de enfermedades infecciosas exóticas, y vigilancia de potenciales vectores autóctonos de dichas enfermedades", en septiembre de 2013 se detecta por primera vez la presencia de Aedes albopictus (Diptera, Culicidae) en la provincia de Valencia en las localidades costeras de Sueca, Cullera y Gandía, gracias a la utilización de trampas de oviposición.

Palabras clave: Mosquito tigre, Culicidae, Vigilancia entomológica, Valencia, España.

\begin{abstract}
First findings of Aedes (Stegomyia) albopictus (Skuse, 1894) in the province of Valencia, Spain

Framed within the project "Entomological surveillance at airports and ports against vectors of imported infectious diseases, and surveillance of potential native vectors of these diseases", on September 2011 Aedes albopictus (Diptera, Culicidae) is reported for the first time in the province of Valencia in the coastal municipalities of Sueca, Cullera and Gandía, using oviposition traps.
\end{abstract}

Key words: Asian tiger mosquito, Culicidae, Entomological surveillance, Valencia, Spain.
Aedes albopictus, comúnmente conocido como mosquito tigre, es un culícido invasor procedente del sudeste asiático que se ha dispersado rápidamente por los cinco continentes en las últimas tres décadas, aprovechando diferentes vías de transporte intercontinental. Tanto es así que, en la actualidad, este mosquito está considerado como una de las cien especies exóticas invasoras más dañinas del mundo (Lowe et al. 2000). Desde su primer hallazgo en Europa en el año 1979 en Albania (Adhami \& Reiter 1998), el nematócero se ha diseminado y establecido por un total de 14 países europeos y dos ciudades-estado (ECDC 2012), casi todos ellos circunscritos a la cuenca 
del mar Mediterráneo,donde se ha adaptado a este clima mediterráneo caracterizado por inviernos templados y lluviosos y veranos secos y calurosos, con otoños y primaveras variables.

Este culícido, aunque en origen circunscrito al ambiente selvático en el sudeste asiático, se encuentra actualmente ligado a ambientes urbanos y periurbanos en diversas partes del globo, por lo que ha devenido en una especie de un marcado carácter sinantrópico. Sus hábitos alimentarios son diurnos de biorritmo bimodal y presenta una marcada antropofilia, aunque la disponibilidad de huéspedes condiciona esta conducta y pueden tomar sangre de una amplia variedad de mamíferos y aves (Richards et al. 2006). Su picadura produce lesiones con fuerte componente inflamatorio y prurito (Giménez et al. 2007), originando, en ocasiones, reacciones alérgicas graves (Montini et al. 2003). Por este motivo, además causan un perjuicio económico de gran impacto a nivel turístico y en actividades al aire libre (Roiz et al. 2007a). No obstante, el principal interés sanitario de este mosquito se centra en su elevada capacidad para transmitir agentes patógenos responsables de enfermedades vectoriales de gran importancia a nivel mundial. Ae. albopictus es capaz de vehiculizar algunas enfermedades parasitarias pero, sobre todo, numerosas virosis, entre las que destacan el dengue, la fiebre amarilla, el virus del Nilo Occidental o el chikungunya (Mitchell 1995). A este respecto, la expansión de Ae. albopictus por Europa ha sido responsable de los brotes autóctonos de chikungunya acontecidos en Italia (región de Emilia-Romaña), en 2007 (Angelini et al. 2007) y Francia (Costa Azul), en 2010 (Grandadam et al. 2011); de dengue, en 2010, en Francia (Costa Azul) (La Ruche et al. 2010) y Croacia (península de Pelješac) (Gjenero-Margan et al. 2011).

El mosquito tigre se detectó por primera vez en España en agosto de 2004, concretamente en el municipio barcelonés de Sant Cugat del Vallès (Aranda et al. 2006). En, aproximadamente, una década, se ha extendido progresivamente desde el norte de Cataluña hasta la Región de Murcia (Collantes \& Delgado 2011), pasando por las provincias valencianas de Alicante (Roiz et al. 2007b, Delacour et al. 2009, Bueno et al. 2010) y Castellón (Delacour Estrella et al. 2010), llegando inclusive a las Islas Baleares (Miquel et al. 2013). Si bien cabe destacar el papel central que han desempeñado los Centros de Atención Primaria del Sistema Nacional de Salud en la rápida detección de la llegada de esta especie, donde el importante aumento de las consultas médicas por reacciones a picaduras de insecto ha posibilitado un estudio más detallado del problema, es el uso de trampas de oviposición u ovitrampas el elemento más eficaz en el ámbito de la vigilancia ambiental del mosquito (CIPCMTC 2011). Estos dispositivos son instrumentos de gran utilidad en los programas de vigilancia de ciertas especies de mosquitos (Fay \& Eliason 1966), particularmente de aquellos cuyos estadios larvales se desarrollan en contenedores artificiales, cavidades de árboles o charcos entre las rocas (Becker et al. 2003), ya que mimetizan los hábitats naturales de cría, constituyendo así un método indirecto de evaluar la presencia de poblaciones de Ae. albopictus (Di Luca et al. 2001).

La Universidad de Zaragoza, en colaboración con el Instituto de Salud Carlos III de Madrid y la Universidad de Murcia, lleva a cabo el trabajo técnico sobre "Vigilancia entomológica en aeropuertos y puertos frente a vectores importados de enfermedades infecciosas exóticas, y vigilancia de potenciales vectores autóctonos de dichas enfermedades", una actividad promovida y financiada por el Ministerio de Sanidad, Servicios Sociales e Igualdad del Reino de España. El establecimiento y la gran expansión del vector observada durante los últimos años en la zona central del litoral castellonense, así como en el sur de la provincia de Alicante (MSSI 2013), hacían temer por la inminente colonización de la provincia de Valencia. En el transcurso del trabajo de vigilancia entomológica del ejercicio de 2013 se dispuso un amplio entramado de trampas de oviposición por municipios litorales, de marcado carácter turístico, pertenecientes a la provincia de Valencia. La disposición de las mismas sigue la línea marcada por los ejes viarios que conforman la Autopista del Mediterráneo, AP-7, y la carretera N-332. Las ovitrampas se situaron en las proximidades de núcleos poblacionales, en lugares susceptibles de albergar al mosquito adulto teniendo en cuenta las preferencias ecológicas de la especie como ser lugares umbríos, con abundante cobertura vegetal y alto grado de humedad. Se procuró que estuvieran resguardadas para evitar su manipulación por parte de cualquier persona ajena a la investigación. En su mayoría se escogieron jardines y parques urbanos, rotondas ajardinadas, zonas residenciales, urbanizaciones y cementerios.

Los análisis desprendidos del estudio de las 
ovitrampas colocadas durante la segunda quincena de septiembre mostraron resultados positivos en las localidades de Sueca, Cullera (ambas pertenecientes a la comarca de la Ribera Baja) y Gandía (comarca de La Safor) (Tabla 1). En Sueca, se colocaron 20 trampas de oviposición, obteniéndose resultados positivos para huevos en dos de ellas, situadas en un jardín urbano adyacente a la Avenida Mestre Serrano y una rotonda en la salida norte de la N-332 en dirección a Valencia. En Cullera, se dispusieron 20 trampas, con cuatro resultados positivos, tres de ellos en el Parque Ausiàs March y otro más en la Plaza de San Isidro, en pleno centro urbano y en las cercanías del Club Náutico de Cullera. En Gandía, se colocaron 25 trampas. Fueron positivas, con puesta de huevos, una situada en la zona de El Grau, en las inmediaciones de la UPV- Campus de Gandía, y otra en la zona residencial Grau i Platja. El material biológico colectado fue eclosionado siguiendo el método de cría en laboratorio descrito por Alarcón-Elbal et al. (2010) y los ejemplares estudiados, tanto larvas como adultos, fueron identificados positivamente como Ae. albopictus mediante las claves de Schaffner et al. (2001). Los ejemplares se encuentran depositados en la colección entomológica de la Unidad de Parasitología y Enfermedades Parasitarias, del Departamento de Sanidad Animal de la Universidad de Zaragoza.

El hecho de haberse detectado previamente en la provincia de Alicante, lugar más alejado de los focos iniciales de la Península Ibérica (Roiz et al. 2007b), o la reciente aparición en las Islas Baleares (Miquel et al. 2013), parecen confirmar que Ae. albopicus realiza más dispersiones artificiales, aprovechando los transportes antrópicos, que naturales, ya que su capacidad de vuelo es reducida. De hecho, se especula que la extensión desde Cataluña hacia el sur se haya realizado a través de las carreteras que recorren la costa mediterránea (MSSI, 2013). Como apuntan Delacour-Estrella et al. (2010), al reportar la primera cita para Castellón, la gran cantidad de desplazamientos de personas, que acuden desde muy diversos lugares hacia estas ciudades costeras, eminentemente turísticas, podría haber facilitado que ejemplares de este insecto fuesen transportados en los vehículos desde zonas con poblaciones estables preexistentes. Tanto es así, que ya hay quien ha bautizado a este díptero con el sobrenombre de "insecto autoestopista", por su especial tendencia a desplazarse vía terrestre aprovechando la actividad comercial y turística (Roiz et al. 2007a), utilizando los automóviles. Este parece ser el caso de su introducción en España (Aranda et al. 2006) y en otros países como Suiza (Flacio et al. 2004) y Alemania (Pluskota et al. 2008). En otros casos, vía marítima, como en Grecia (Samanidou-Voyadjoglou et al. 2005). Sin embargo, el transporte accidental de fases inmaduras en recipientes también ha resultado eficaz en muchos casos. Ha sido trascendental el hallazgo de huevos en cargamentos de neumáticos usados, transportados largas distancias. Fue la vía de entrada en Italia (Sabatini et al. 1990), Francia (Schaffner \& Karch 2000) o Bélgica (Schaffner et al. 2004). Otra vía similar es el transporte de productos de jardinería, como los tronquitos de bambú de la suerte (Dracaena spp. Vand. ex L.), medio por el cual se introdujo en Holanda (Scholte et al. 2007).

Lejos de detenerse, y tras una década desde su introducción en el país, la incuestionable expansión del Ae. albopictus por el este peninsular es un problema patente e ineludible. Contrasta esta incuestionable realidad con los modelos proporcionados por diversos autores (Collantes et al., en prensa) que señalan bajos niveles de riesgo en numerosas áreas del este peninsular que ya han ido, o están siendo, colonizadas. En nuestra opinión, gran parte del territorio español presenta unas condiciones ecológicas, que no climáticas, muy favorables para una colonización masiva del vector. En las áreas teóricamente menos óptimas, según los mapas de riesgo más recientes, existen zonas urbanizadas y periurbanas que pueden proporcionar hábitats adecuados per se (Collantes et al., en prensa). La literatura científica es desbordante en dos vías de afluencia para la resolución de este problema. La primera es involucrar a la población local en los planes de lucha contra el mosquito, es decir, la participación ciudadana. En este sentido, la medida más efectiva para la de prevención y control de este aedino es la eliminación mecánica de los lugares potenciales de cría del mosquito, por lo que las acciones de sensibilización poblacional son el elemento clave (CIPCMTC 2011). En segundo término, el empleo conjunto de todas las herramientas de lucha reactiva. La aplicación de medidas de control integrado parece ser la mejor opción. La estrategia de control se debe basar en la utilización de productos larvicidas y, sólo en casos estrictamente necesarios y justificados desde el punto de vista socio-sanitario, realizar tratamientos contra los mosquitos adultos. 


\begin{tabular}{|c|c|c|c|c|c|c|}
\hline & Localización & Trampas & Coordenadas & Positivos & No huevos & Eclosión \\
\hline \multirow{6}{*}{ 选 } & Parque Ausiàs March & G1-G5 & & - & & \\
\hline & Rotonda Paseeig Germaníes & G6-G10 & & - & & \\
\hline & \multirow{2}{*}{ Rotonda UPV - Campus Gandía } & G11-G15 & & 1 & & \\
\hline & & G14 & $38^{\circ} 59^{\prime} 43.85^{\prime \prime} \mathrm{N} \mathrm{0} 10^{\prime} 2.29^{\prime \prime O}$ & + & 34 & + \\
\hline & \multirow{2}{*}{ Parque C/. Castella-Lleó } & G16-20 & & 1 & & \\
\hline & & G20 & $39^{\circ} 0^{\prime} 49.40^{\prime \prime} \mathrm{N} 0^{\circ} 10^{\prime} 33.63^{\prime \prime} \mathrm{O}$ & + & 26 & + \\
\hline \multirow{6}{*}{ 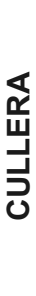 } & \multirow{3}{*}{ Parque Camino Molí Cremat } & C1-C10 & & 2 & & \\
\hline & & $\mathrm{C} 6$ & $39^{\circ} 9^{\prime} 41.67 " \mathrm{~N} 0^{\circ} 15^{\prime} 27.97 " \mathrm{O}$ & + & 13 & + \\
\hline & & $\mathrm{C9}$ & $39^{\circ} 9^{\prime} 40.81 " \mathrm{~N} 0^{\circ} 15^{\prime} 29.11 " \mathrm{O}$ & + & 54 & + \\
\hline & \multirow[t]{2}{*}{ Parque Plaza San Isidre - Correos } & C11-C15 & & 1 & & \\
\hline & & C11 & $39^{\circ} 9^{\prime} 47.40^{\prime \prime} \mathrm{N} 0^{\circ} 15^{\prime} 24.64 " \mathrm{O}$ & + & 7 & + \\
\hline & Rotonda C/. Mercedes Marí Domínguez & C16-C20 & & - & & \\
\hline \multirow{6}{*}{ 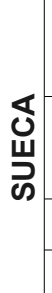 } & \multirow{2}{*}{ Jardín Av. Mestre Serrano } & S1-S5 & & 1 & & \\
\hline & & S1 & $39^{\circ} 12^{\prime} 4.55^{\prime \prime} 0^{\circ} 18^{\prime} 57.53 " \mathrm{O}$ & + & 14 & + \\
\hline & \multirow{2}{*}{ Rotonda Av. Gandient - N332 } & S6-S10 & & 1 & & \\
\hline & & S9 & $39^{\circ} 12^{\prime} 17.72^{\prime \prime} \mathrm{N} \mathrm{0} 19^{\prime} 4.67 " \mathrm{O}$ & + & 3 & + \\
\hline & Jardines Av. del Mar - CV500 & S11-S15 & & - & & \\
\hline & Parque Paseo l'Estació & S16-S20 & & - & & \\
\hline
\end{tabular}

Tabla 1. Localización de las trampas de oviposición colocadas en los términos municipales de Gandía, Cullera y Sueca. Se detallan las coordenadas de los resultados positivos a la presencia de huevos, así su número y eclosión en laboratorio de ejemplares de Ae. albopictus. Table 1. Location of ovitraps placed in the municipalities of Gandía, Cullera and Sueca. For positive traps, coordinates, number of eggs and eclosion in laboratory are detailed

\section{Agradecimientos}

El presente trabajo está subvencionado por el Ministerio de Sanidad, Servicios Sociales e Igualdad. Asimismo, los autores desean mostrar su más sincero agradecimiento a la empresa de servicios de control de plagas y sanidad ambiental Compañía de Tratamientos de Levante S.L. (CTL), por su participación en la realización de los muestreos.

\section{Referencias}

Adhami JR \& Reiter P. 1998. Introduction and establishment of Aedes (Stegomyia) albopictus Skuse (Diptera: Culicidae) in Albania. Journal of American Mosquito Control Association 14: 340-343.

Alarcón-Elbal PM, Delacour S, Pinal R, Ruiz-Arrondo I, Muñoz A, Bengoa M, Eritja R \& Lucientes J 2010. Establecimiento y mantenimiento de una colonia autóctona española de Aedes (Stegomyia) albopictus Skuse, 1894, (Diptera, Culicidae) en laboratorio. Revista Ibero-Latinoamericana de Parasitología 69(2): 140-148.

Angelini R, Finarelli AC, Angelini P, Po C, Petropulacos $K$, Macini $P$, Fiorentini C, Fortuna C, Venturi G, Romi R, Majori G, Nicoletti L, Rezza G \& Cassone A.
2007. An outbreak of chikungunya fever in the province of Ravenna, Italy. Eurosurveillance 12(36): 3260. Disponible en Internet en: http://www.eurosur veillance. org/ViewArticle. aspx?Articleld=3260 [accedido el 1 de octubre de 2013]

Aranda C, Eritja R \& Roiz D. 2006. First record and stablishment of the mosquito Aedes albopictus in Spain. Medical and Veterinary Entomology 20: 150152.

Becker N, Petric D, Zgomba M, Boase C, Dahl C, Lane J \& Kaiser A. 2003. Mosquitoes and their control. Kluwer Academic/ Plenum Publishers New York. 498 pp.

Bueno-Marí R, Chordá Olmos FA, Barnués-Bañeres A \& Jiménez-Peydró R. 2010. Detección de Aedes albopictus (Skuse, 1894) en Torrevieja (Alicante, España). Boletín de la Asociación española de Entomología 33 (3-4): 529-532.

Collantes F \& Delgado JA. 2011. Primera cita de Aedes (Stegomyia) albopictus (Skuse, 1894) en la Región de Murcia. Anales de Biología 33: 99-101.

Collantes F, Delgado JA, Alarcón-Elbal P, Delacour S \& Lucientes J. (en prensa). First confirmed outdoor winter reproductive activity of Asian tiger mosquito in Europe. Journal of Vector Ecology. (aceptado 29-ix2013)

Comissió Interinstitucional per a la Prevenció i Control del Mosquit Tigre a Catalunya (CIPCMTC). 2011. Estratègia per a la prevenció i el control del mosquit 
tigre a Catalunya. Disponible en Internet en: http://www20.gencat.cat/docs/canalsalut/Home $\% 20$ Canal\%20Salut/Ciutadania/Vida_saludable/Entorn_ saludable/el_medi/les_plagues/el_mosquit_tigre/Doc uments/estrategia_mosquit_tigre_10_juny_2011.pdf [accedido el 1 de octubre de 2013]

Delacour S, Alarcón-Elbal P, Bengoa M, Melero-Alcíbar R, Pinal R, Ruiz-Arrondo I, Molina R \& Lucientes J. 2009. Aedes (Stegomyia) albopictus (Skuse, 1894) primera cita en Torrevieja (Alicante). Boletín Sociedad Entomológica Aragonesa 45: 518.

Delacour-Estrella $S$, Bravo-Minguet $D$, Alarcón-Elbal PM, Bengoa M, Casanova A, Melero-Alcibar R, Pinal R, Ruiz-Arrondo I, Molina R \& Lucientes J. (2010) Detección de Aedes (Stegomyia) albopictus (Skuse, 1894) (Diptera: Culicidae) en Benicàssim. Primera cita para la provincia de Castellón (España). Boletín de la Sociedad Entomológica Aragonesa 47: 440.

Di Luca M, Toma L, Severini F, D'Ancona F \& Romi R. 2001. Aedes albopictus a Roma: monitoraggio nel trieninio 1998-2000. Annali dell Istituto Superiore di Sanita 37: 249-254.

European Centre for Disease Prevention and Control (ECDC). 2012. Guidelines for the surveillance of invasive mosquitoes in Europe. Disponible en Internet en: http://ecdc.europa.eu/en/publications/ publications/ter-mosquito-surveillance-guidelines.pdf [accedido el 1 de octubre de 2013]

Fay RW \& Eliason DA. 1966. A preferred oviposition site as a surveillance method for Aedes aegypti. Mosquito News 26: 531-535.

Flacio E, Lüthy P, Patocchi N, Guidotti F, Tonolla M \& Peduzzi R. 2004. Primo ritrovamento di Aedes albopictus in Svizzera. Bolletino della Società ticinese di Scienze naturali 92:141-142.

Giménez N, Barahona $M$, Casasa A, Domingo A, Gavagnach M \& Marti C. 2007. Llegada de Aedes albopictus a España, un nuevo reto para la salud pública. Gaceta Sanitaria 21(1): 25-28.

Gjenero-Margan I, Aleraj B, Krajcar D, Lesnikar V, Klobučar A, Pem-Novosel I, Kurečić-Filipović S, Komparak S, Martić R, Đuričić S, Betica-Radić L, Okmadžić J, Vilibić-Čavlek T, Babić-Erceg A, Turković B, Avšić-Županc T, Radić I, Ljubić M, Šarac K, Benić N \& Mlinarić-Galinović G. 2011. Autochthonous dengue fever in Croatia, August-September 2010. Eurosurveillance 16(9):pii=19805. Disponible en Internet en: <http://www.eurosurveillance.org/images/dynamic/EE/V16N09/ art19805.pdf [accedido el 1 de octubre de 2013]

Grandadam M, Caro V, Plumet S, Thiberge JM, Souarès $Y$, Failloux $A B$, Tolou HJ, Budelot M, Cosserat D, Leparc-Goffart I \& Desprès P. 2011. Chikungunya virus, southeastern France. Emerging Infectious Diseases 17(5): 910-913.

La Ruche G, Souarès Y, Armengaud A, Peloux-Petiot F, Delaunay $P$, Desprès $P$, Lenglet $A$, Jourdain $F$, Leparc-Goffart I, Charlet F, Ollier L, Mantey K, Mollet T, Fournier JP, Torrents R, Leitmeyer K, Hilairet $\mathrm{P}$, Zeller $\mathrm{H}$, Van Bortel W, Dejour-Salamanca D,
Grandadam M \& Gastellu-Etchegorry M. 2010. First two autochthonous dengue virus infections in metropolitan France, September 2010. Eurosurveillance 15(39): 19676. Disponible en Internet en: http:// www.eurosurveillance.org/images/dynamic/EE/V15N 39/art19676.pdf [accedido el 1 de octubre de 2013]

Lowe S, Browne M, Boudjelas S \& de Poorter M. 2000. 100 of the world's worst invasive alien species: A selection from the Global Invasive Species Database. Auckland: IUCN/SSC Invasive Species Specialist Group (ISSG). Disponible en Internet en: http:// www.issg.org/database/species/reference_files/100 English.pdf [accedido el 1 de octubre de 2013]

Ministerio de Sanidad, Servicios Sociales e Igualdad (MSSI). 2013. Evaluación del riesgo de introducción y circulación del virus de dengue en España. Mayo 2013. Disponible en Internet en: http://www.msssi. gob.es/profesionales/saludPublica/ccayes/analisisitu acion/doc/evRiDe_5_11.pdf [accedido el 1 de octubre de 2013]

Miquel M, del Río R, Borràs D, Barceló $C$, Paredes Esquivel C, Lucientes J \& Miranda MA. 2013. First detection of Aedes albopictus (Diptera: Culicidae) in the Balearic Islands (Spain) and assessment of its establishment according to the ECDC guidelines. Journal of the European Mosquito Control Association 31: 8-11.

Mitchell CJ. 1995. Geographic spread of Aedes albopictus and potential for involvement in arbovirus cycles in the Mediterranean basin. Journal of Vector Ecology 20:44-58.

Montini L. Conti G, Pennisi MA, Di Nardo M \& Antonelli M 2003. Acute respiratory failure after a tigre mosquito bite: a case report. Intensive Care Medicine 29: 1026-1027.

Pluskota B, Storch V, Braunbeck T, Beck M \& Becker N. 2008. First record of Stegomyia albopicta (Skuse) (Diptera: Culicidae) in Germany. European Mosquito Bulletin 26: 1-5.

Richards SL, Ponnusamy L, Unnasch TR, Hassan HK \& Apperson CS. 2006. Host-feeding patterns of Aedes albopictus (Diptera: Culicidae) in relation to availability of human and domestic animals in suburban landscapes of Central North Carolina. Journal of Medical Entomology 43(3): 543-551.

Roiz D, Eritja R, Melero-Alcibar R, Molina R, Marquès E, Ruiz S, Escosa R, Aranda C. \& Lucientes J. 2007a. Distribución de Aedes (Stegomyia) albopictus (Skuse, 1894) (Diptera, Culicidae) en España. Boletín Sociedad Entomológica Aragonesa 40: 523526.

Roiz D, Eritja R, Escosa R, Lucientes J, Marquès E, Melero-Alcíbar R, Ruiz S. \& Molina R. 2007b. A survey of mosquitoes breeding in used tires in Spain for the detection of imported potential vector species. Journal of Vector Ecology 32 (1): 10-15.

Sabatini A, Ranieri V, Trovato G \& Coluzzi M. 1990. Aedes albopictus in Italia e posible diffusione della specie nell'area mediterranea. Parassitologia 32: 301-304. 